\title{
Multifractal formalism of an inhomogeneous multinomial measure with various parameters
}

\author{
A. SAMti \\ Analysis, Probability 8 Fractals Laboratory LR18ES17 \\ University of Monastir, Faculty of Sciences of Monastir \\ Department of Mathematics, 5019-Monastir, Tunisia \\ amal_samti@yahoo.fr
}

Received May 18, 2020

Presented by Mostafa Mbekhta

Accepted July 7, 2020

Abstract: In this paper, we study the refined multifractal formalism in a product symbolic space and we estimate the spectrum of a class of inhomogeneous multinomial measures constructed on the product symbolic space.

Key words: Hausdorff dimension, packing dimension, fractal, multifractal.

AMS Subject Class. (2010): 28A80, 28A78, 28A12, 11K55.

\section{INTRODUCTION}

The multifractal formalism of a measure $\mu$ aims to establish a relationship between the dimension of level set of the local Hölder exponent of $\mu$ to the Legendre transform of what is called the "free energy" function. A problem initially raised and studied for physical motivations [8, 9, 11, 12, 10]. It will be convenient to give a brief description of the multifractal formalism. Let $\mathbb{X}$ be a metric space. The local Hölder exponent $\alpha_{\mu}(x)$ at the point $x \in \mathbb{X}$ is defined to be

$$
\alpha_{\mu}(x)=\lim _{r \rightarrow 0} \frac{\log \mu(B(x, r))}{\log r}
$$

where $B(x, r)$ stands for the ball of radius $r$ centered at $x$. The measure $\mu$ is said to satisfy the multifractal formalism at $\alpha \geq 0$, if the Hausdorff dimension $(\operatorname{dim})$ and the packing dimension (Dim) of the level set $E(\alpha)$ which is defined by

$$
E(\alpha)=\left\{x \in \operatorname{supp}(\mu): \alpha_{\mu}(x)=\alpha\right\},
$$

are equal respectively to the value of the Legendre transform at $\alpha$ of a scale function $\tau_{\mu}$ associated to the measure $\mu$, i.e.,

$$
\operatorname{dim} E(\alpha)=\operatorname{Dim} E(\alpha)=\tau_{\mu}^{*}(\alpha)
$$


where $f^{*}(x)=\inf _{y}(x y+f(y))$ is the Legendre transform of a function $f$ and $\operatorname{supp}(\mu)$ is the topological support of $\mu$.

The upper bound for $\operatorname{dim} E(\alpha)$ (respectively $\operatorname{Dim} E(\alpha)$ ) is obtained by a standard covering argument as Besicovisch's covering Theorem and Vitali's Lemma [13. However, the lower bound is usually much harder to prove, it is related to the existence of an auxiliary measure such as a Gibbs measure [13. or a Frostman measure [3] which is supported by the set to be analyzed.

For this reason, F. Ben Nasr et al. [4 improved the Olsen's result in describing a class of measures satisfying the multifractal formalism and proposed a new sufficient condition that gives the lower bound. In such a situation, they concluded that $B_{\mu}(q)=b_{\mu}(q)$, where $b_{\mu}$ and $B_{\mu}$ are Olsen's functions. Besides, they constructed inhomogeneous Bernoulli products, such measures whose both multifractal dimension functions $b_{\mu}$ and $B_{\mu}$ agree at one or two points only. Which implies a valid refined multifractal formalism no more than two points. In [5], Ben Nasr and Peyrière constructed an example of a "bad" measure on the interval $\{0,1\}^{\mathbb{N}}$ for which the Olsen's functions $b_{\mu}$ and $B_{\mu}$ differ and the Hausdorff dimensions of the sets $E(\alpha)$ are given by the Legendre transform of $b_{\mu}$, and their packing dimensions by the Legendre transform of $B_{\mu}$, i.e., $b_{\mu}(q)<B_{\mu}(q)$ for all $q \in\{0,1\}$ and

$$
\operatorname{dim} E(\alpha)=b_{\mu}^{*}(\alpha) \text { and } \operatorname{Dim} E(\alpha)=B_{\mu}^{*}(\alpha), \quad \text { for some } \alpha \geq 0 .
$$

Shen [14] and $\mathrm{Wu}$ et al. [17, 18, 19] revisited this example such that the functions $B_{\mu}$ and $b_{\mu}$ can be real analytic. Motivated by these examples N. Attia and B. Selmi [1, 2] introduced and studied a new multifractal formalism based on the Hewitt-Stromberg measures and showed that this formalism is completely parallel to Olsen's multifractal formalism based on the Hausdorff and packing measures.

In the present work, let $2 \leq r_{1}<r_{2}$ be two integers, we consider a class of measures defined on a product symbolic space $\mathbb{A}_{1} \times \mathbb{A}_{2}$ endowed with the distance product where $\mathbb{A}_{i}=\left\{0, \ldots, r_{i}-1\right\}$ for $i=1,2$, and constructed on the rectangles that flatten as their diameters tend to zero. However, these rectangles do not allow the calculation of the Hausdorff dimension, hence the difficulty of the problem. The aim of this paper is to study the validity of the refined multifractal formalism of this class of measures.

The paper is organized as follows. In Section 2, we give some notations and definitions which will be useful. In the third section we consider a sequence of finite partitions of a product symbolic space made of rectangles and we show through an example that the almost squares allow the calculation of the 
Hausdorff and packing dimensions. In Section 4, we consider a variant of the refined multifractal formalism as already introduced by Ben Nasr and Peyrière [5] which we adapt it to almost squares and estimate the dimensions of the level sets $E(\alpha)$. Finally, we apply our results to a class of inhomogeneous measures defined on the product symbolic space.

\section{Notations AND DEFinitions}

In this section, we will recall the Hausdorff and packing measures and their dimensions. Let $(\mathbb{X}, d)$ be a separable metric space. The diameter of a non-empty set $E \subseteq \mathbb{X}$ is given by

$$
\operatorname{diam} E=\sup \{d(x, y): x, y \in E\},
$$

with the convention that $\operatorname{diam}(\emptyset)=0$.

We define the closed ball with center $x \in \mathbb{X}$ and radius $r>0$ as

$$
B(x, r)=\{y \in \mathbb{X}: d(x, y) \leq r\} .
$$

A finite or countable collection of subsets $\left\{U_{i}\right\}_{i}$ of $\mathbb{X}$ is called a $\delta$-cover of $E \subseteq \mathbb{X}$, if for each $i$ we have $\operatorname{diam} U_{i} \leq \delta$ and $E \subset \bigcup_{i} U_{i}$.

Suppose that $E$ is a subset of $\mathbb{X}$ and $s$ is a non-negative number. For any $\delta>0$ we define

$$
\mathcal{H}_{\delta}^{s}(E)=\inf \left\{\sum_{i} \operatorname{diam}\left(U_{i}\right)^{s}:\left\{U_{i}\right\}_{i} \text { is a } \delta \text {-cover of } E\right\} .
$$

As $\delta$ decreases, the class of $\delta$-covers of $E$ is reduced. Therefore, this infimum increases and approaches a limit as $\delta \searrow 0$. Thus we define

$$
\mathcal{H}^{s}(E)=\lim _{\delta \rightarrow 0} \mathcal{H}_{\delta}^{s}(E)
$$

We term $\mathcal{H}^{s}(E)$ the $s$-dimensional Hausdorff measure of $E$. Then we define the Hausdorff dimension of $E$ as

$$
\operatorname{dim}(E)=\sup \left\{s \geq 0: \mathcal{H}^{s}(E)=\infty\right\}=\inf \left\{s \geq 0: \mathcal{H}^{s}(E)=0\right\} .
$$

Remark 1. Notice that the covering of $E$ with centered balls in $E$ allow the calculation of the Hausdorff dimension of $E$, for more details see [7]. 
We will now define the packing measure. First, let define a $\delta$-packing of $E \subset \mathbb{X}$ to be a finite or countable collection of disjoint balls $\left\{B\left(x_{i}, r_{i}\right)\right\}_{i}$ of diameter at most $\delta$ and with centers in $E$. For $s \geq 0$ and $\delta>0$, let

$$
\overline{\mathcal{P}}_{\delta}^{s}(E)=\sup \left\{\sum_{i}\left(2 r_{i}\right)^{s}:\left\{B\left(x_{i}, r_{i}\right)\right\}_{i} \text { is a } \delta \text {-packing of } E\right\} .
$$

From this the $s$-dimensional pre-packing measure $\overline{\mathcal{P}}^{s}$ of $E$ is defined by

$$
\overline{\mathcal{P}}^{s}(E)=\lim _{\delta \rightarrow 0} \overline{\mathcal{P}}_{\delta}^{s}(E)
$$

Finally, we define the $s$-dimensional packing measure $\mathcal{P}^{s}(E)$ of $E$ by

$$
\mathcal{P}^{s}(E)=\inf \left\{\sum_{i} \overline{\mathcal{P}}^{s}\left(E_{i}\right): E \subset \bigcup_{i=1}^{\infty} E_{i}\right\} .
$$

The packing dimension of $E$, denoted by $\operatorname{Dim}(E)$, is defined in the same way as Hausdorff dimension, that means

$$
\operatorname{Dim}(E)=\sup \left\{s \geq 0: \mathcal{P}^{s}(E)=\infty\right\}=\inf \left\{s \geq 0: \mathcal{P}^{s}(E)=0\right\} .
$$

For more details about the Hausdorff, packing measures and their dimensions see [15, 16, 7].

\section{Calculation of the Hausdorff and packing dimensions on the PRODUCT SYMBOLIC SPACE ON DIFFERENT BASIS}

For practical reasons, we shall need basic notions about the set of words on an alphabet. Let $2 \leq r_{1}<r_{2}$ be two integers. For $i \in\{1,2\}$, given $A_{i}=\left\{0, \ldots, r_{i}-1\right\}$ a finite alphabet. For all $n \in \mathbb{N}^{*}$, each element in $A_{i}^{n}$ is denoted by a string of $n$ letters or digits in $A_{i}$ that we call a word; by convention $A_{i}^{0}$ is reduced to the empty word $\emptyset$. Let $A_{i}^{*}=\bigcup_{n \geq 0} A_{i}^{n}$ be the set of finite words built over $A_{i}$ and $\mathbb{A}_{i}=A_{i}^{\mathbb{N}^{*}}$ the symbolic space over $A_{i}$.

The set $A_{i}^{*} \cup \mathbb{A}_{i}$ is endowed with the concatenation operation: If $\omega \in A_{i}^{*}$ and $\omega^{\prime} \in A_{i}^{*} \cup \mathbb{A}_{i}$, we denote by $\omega \cdot \omega^{\prime}$ the word obtained by juxtaposition of the two words $\omega$ and $\omega^{\prime}$.

For each finite word $\omega \in A_{i}^{*},[\omega]$ is the cylinder $\omega \cdot \mathbb{A}_{i}=\left\{\omega \cdot \omega^{\prime}: \omega^{\prime} \in \mathbb{A}_{i}\right\}$. Furthermore, if $\omega=\omega_{1} \cdots \omega_{k} \cdots \in \mathbb{A}_{i}$ and $n \in \mathbb{N}$ then $\omega_{\mid n}$ stands for the prefix $\omega_{1} \cdots \omega_{n}$ of $\omega$ for $n \geq 1$ and the empty word otherwise. Each set $\mathbb{A}_{i}$ 
is endowed with the ultrametric distance $d_{i}:\left(z, z^{\prime}\right) \in \mathbb{A}_{i}^{2} \longmapsto r_{i}^{-\left|z \wedge z^{\prime}\right|}$, where $z \wedge z^{\prime}$ is defined to be the longest prefix common to both $z$ and $z^{\prime}$ and $|z|$ the length of a word $z \in A_{i}^{*} \cup \mathbb{A}_{i}$. Then the product symbolic space $\mathbb{A}_{1} \times \mathbb{A}_{2}$ is endowed with the ultrametric distance.

$$
d\left((x, y),\left(x^{\prime}, y^{\prime}\right)\right)=\max \left(d_{1}\left(x, x^{\prime}\right), d_{2}\left(y, y^{\prime}\right)\right)
$$

In the next, if $\omega \in A_{1}^{k}$ and $\omega^{\prime} \in A_{2}^{k^{\prime}}$, we call $R\left(\omega, \omega^{\prime}\right)$ the rectangle obtained as the product of the cylinders $[\omega]$ and $\left[\omega^{\prime}\right]$. We denote by

$$
\left|R\left(\omega, \omega^{\prime}\right)\right|_{M}=\sup \left(\frac{1}{r_{1}^{k}}, \frac{1}{r_{2}^{k^{\prime}}}\right)
$$

and

$$
\left|R\left(\omega, \omega^{\prime}\right)\right|_{m}=\inf \left(\frac{1}{r_{1}^{k}}, \frac{1}{r_{2}^{k^{\prime}}}\right)
$$

We say that a sequence $\left\{\xi_{n}\right\}_{n>1}$ of finite partitions of $\mathbb{A}_{1} \times \mathbb{A}_{2}$ made of rectangles satisfies condition (1) if

$$
\lim _{n \rightarrow \infty} \sup _{R \in \xi_{n}} \operatorname{diam}(R)=0 \quad \text { and } \quad \xi_{n+1} \quad \text { is a refinement of } \xi_{n} .
$$

In all over this work, we will consider a sequence $\left\{\xi_{n}\right\}_{n>1}$ of finite partitions of $\mathbb{A}_{1} \times \mathbb{A}_{2}$ made of rectangles verifying (1) and we put $\xi=\bigcup_{n>1} \xi_{n}$. If $R$ belongs to $\xi_{n+1}$, we define by $p(R)$ the element of $\xi_{n}$ that contains it.

Let $E$ be a nonempty subset of $\mathbb{A}_{1} \times \mathbb{A}_{2}$ and $s$ a strictly positive real number. For all $\varepsilon>0$, a finite or countable collection of rectangles $\left\{R_{j}\right\}_{j}$ is called an $\varepsilon$-covering of $E$ if $\operatorname{diam}\left(R_{j}\right) \leq \varepsilon$ for all $j$ and $E \subset \bigcup_{j} R_{j}$.

Let

$$
\mathcal{H}_{\xi, \varepsilon}^{s}(E)=\inf \left\{\sum_{j} \operatorname{diam}\left(R_{j}\right)^{s}: R_{j} \in \xi,\left\{R_{j}\right\}_{j} \text { is an } \varepsilon \text {-covering of } E\right\}
$$

and

$$
\mathcal{H}_{\xi}^{s}(E)=\lim _{\varepsilon \rightarrow 0} \mathcal{H}_{\xi, \varepsilon}^{s}(E) .
$$

Finally, the $\operatorname{dimension}_{\operatorname{dim}_{\xi}}(E)$ is given by

$$
\operatorname{dim}_{\xi}(E)=\inf \left\{s>0: \mathcal{H}_{\xi}^{s}(E)=0\right\}=\sup \left\{s>0: \mathcal{H}_{\xi}^{s}(E)=\infty\right\} .
$$


Here, we define an $\varepsilon$-packing of $E \subset \mathbb{A}_{1} \times \mathbb{A}_{2}$ to be a finite or countable collection of disjoint rectangles $\left\{R_{j}\right\}_{j}$ of diameter not exceeding $\varepsilon$ and with $R_{j} \cap E \neq \emptyset$. For $\varepsilon>0$, we define

$$
\overline{\mathcal{P}}_{\xi, \varepsilon}^{s}(E)=\sup \left\{\sum_{j} \operatorname{diam}\left(R_{j}\right)^{s}: R_{j} \in \xi,\left\{R_{j}\right\}_{j} \text { is an } \varepsilon \text {-packing of } E\right\} .
$$

Then $\overline{\mathcal{P}}_{\xi, \varepsilon}^{s}(E)$ decreases as $\varepsilon$ increases, so we may take the limit

$$
\overline{\mathcal{P}}_{\xi}^{s}(E)=\lim _{\varepsilon \rightarrow 0} \overline{\mathcal{P}}_{\xi, \varepsilon}^{s}(E) .
$$

Unfortunately, $\overline{\mathcal{P}}_{\xi}^{s}(E)$ is not an outer measure, to overcome this difficulty we define

$$
\mathcal{P}_{\xi}^{s}(E)=\inf \left\{\sum_{j} \overline{\mathcal{P}}_{\xi}^{s}\left(E_{j}\right): E \subseteq \bigcup_{j} E_{j}\right\} .
$$

The definition of packing dimension parallels that of Hausdorff dimension. So, let $\operatorname{Dim}_{\xi}(E)$ defined such that

$$
\begin{aligned}
\operatorname{Dim}_{\xi}(E) & =\inf \left\{s>0: \mathcal{P}_{\xi}^{s}(E)=0\right\} \\
& =\sup \left\{s>0: \mathcal{P}_{\xi}^{s}(E)=\infty\right\} .
\end{aligned}
$$

In the following proposition we will give some conditions on a family $\xi$ of rectangles of the symbolic space $\mathbb{A}_{1} \times \mathbb{A}_{2}$ such that for every part $E$ of $\mathbb{A}_{1} \times \mathbb{A}_{2}$, we have

$$
\operatorname{dim}(E)=\operatorname{dim}_{\xi}(E) \quad \text { and } \quad \operatorname{Dim}(E)=\operatorname{Dim}_{\xi}(E)
$$

Proposition 3.1. Suppose that

(i) $\lim _{n \rightarrow \infty} \sup _{R \in \xi_{n}} \log |R|_{m} / \log |R|_{M}=1$,

(ii) $\lim _{n \rightarrow \infty} \sup _{R \in \xi_{n}} \log |R|_{M} / \log |p(R)|_{M}=1$.

Then for any part $E$ of $\mathbb{A}_{1} \times \mathbb{A}_{2}$, we have

$$
\begin{aligned}
\operatorname{Dim}_{\xi}(E) & =\operatorname{Dim}(E), \\
\operatorname{dim}_{\xi}(E) & =\operatorname{dim}(E) .
\end{aligned}
$$


Proof. In order to prove the equality (2), we start by proving that $\operatorname{Dim}_{\xi}(E) \leq \operatorname{Dim}(E)$.

Let $t>\operatorname{Dim}(E)$ and $\eta>0$ such that $\frac{t}{1+\eta}>\operatorname{Dim}(E)$. It follows from assumption (i) that there exists an integer $n_{0}$ such that for all $n \geq n_{0}$ and for all $R \in \xi_{n}$, we have

$$
|R|_{M}^{1+\eta} \leq|R|_{m} .
$$

Take $\left\{E_{j}\right\}_{j}$ a cover of $E$ and choose $\left\{R_{k}\right\}_{k}$ an $\varepsilon$-packing of $E_{j}$ with $\varepsilon \leq \inf _{R \in \xi_{n_{0}}} \operatorname{diam}(R)$. For $j \in \mathbb{N}$, fix $x_{k} \in R_{k} \cap E_{j}$, we denote by $B_{k}=$ $B\left(x_{k},\left|R_{k}\right|_{m}\right)$. It is clear that $\left\{B_{k}\right\}_{k}$ is an $\varepsilon$-packing of $E_{j}$.

As $\varepsilon \leq \inf _{R \in \xi_{n_{0}}} \operatorname{diam}(R)$ we get for all integer $k$,

$$
\left|R_{k}\right|_{M}^{1+\eta} \leq\left|R_{k}\right|_{m}
$$

and

$$
\sum_{k} \operatorname{diam}\left(R_{k}\right)^{t} \leq \sum_{k} \operatorname{diam}\left(B_{k}\right)^{\frac{t}{1+\eta}}
$$

Then,

$$
\overline{\mathcal{P}}_{\xi, \varepsilon}^{t}\left(E_{j}\right) \leq \overline{\mathcal{P}}_{\varepsilon}^{\frac{t}{1+\eta}}\left(E_{j}\right)
$$

and as $\varepsilon$ goes to 0 , yields

$$
\overline{\mathcal{P}}_{\xi}^{t}\left(E_{j}\right) \leq \overline{\mathcal{P}}^{\frac{t}{1+\eta}}\left(E_{j}\right)
$$

Therefore,

$$
\mathcal{P}_{\xi}^{t}(E) \leq \mathcal{P}^{\frac{t}{1+\eta}}(E)<+\infty
$$

consequently,

$$
\operatorname{Dim}_{\xi}(E)<t, \text { for all } t>\operatorname{Dim}(E),
$$

which implies that

$$
\operatorname{Dim}_{\xi}(E) \leq \operatorname{Dim}(E)
$$

In order to obtain the other inequality, fix $t>\operatorname{Dim}_{\xi}(E)$ and $\eta>0$ such that $\frac{t}{1+\eta}>\operatorname{Dim}_{\xi}(E)$. Using assumption (ii) there exists an integer $n_{0}$ such that for all $n \geq n_{0}$ and for all $R \in \xi_{n}$, we have

$$
|P(R)|_{M}^{1+\eta} \leq|R|_{M}
$$


Let $\left\{E_{j}\right\}_{j}$ be a cover of $E$ and $\left\{B_{k}=B\left(x_{k}, r_{k}\right)\right\}_{k}$ an $\varepsilon$-packing of $E_{j}$ with $\varepsilon \leq \inf _{R \in \xi_{n_{0}}} \operatorname{diam}(R)$. If $R_{k}$ is a rectangle such that

$$
R_{k} \subset B\left(x_{k}, r_{k}\right) \quad \text { and } \quad P\left(R_{k}\right) \nsubseteq B\left(x_{k}, r_{k}\right) \text {, }
$$

then $\left\{R_{k}\right\}_{k}$ is an $\varepsilon$-packing of $E_{j}$. Since $\varepsilon \leq \inf _{R \in \xi_{n_{0}}} \operatorname{diam}(R)$, we have for all $k \in \mathbb{N}$,

$$
\left|P\left(R_{k}\right)\right|_{M}^{1+\eta} \leq\left|R_{k}\right|_{M} .
$$

Taking into account relations (6) and (7), we have

$$
\sum_{k} \operatorname{diam}\left(B_{k}\right)^{t} \leq \sum_{k} \operatorname{diam}\left(P\left(R_{k}\right)\right)^{t} \leq \sum_{k} \operatorname{diam}\left(R_{k}\right)^{\frac{t}{1+\eta}} .
$$

So,

$$
\overline{\mathcal{P}}_{\varepsilon}^{t}\left(E_{j}\right) \leq \overline{\mathcal{P}}_{\xi, \varepsilon}^{\frac{t}{1+\eta}}\left(E_{j}\right)
$$

As $\varepsilon$ goes to zero,

$$
\overline{\mathcal{P}}^{t}\left(E_{j}\right) \leq \overline{\mathcal{P}}_{\xi}^{\frac{t}{1+\eta}}\left(E_{j}\right)
$$

Then, we obtain

$$
\mathcal{P}^{t}(E) \leq \mathcal{P}_{\xi}^{\frac{t}{1+\eta}}(E)<+\infty
$$

Hence,

$$
\operatorname{Dim}(E) \leq \operatorname{Dim}_{\xi}(E)
$$

which achieves the proof of equality (2).

Now, we will be interested in proving the equality (3).

It is easy to see that $\mathcal{H}^{t}(E) \leq \mathcal{H}_{\xi}^{t}(E)$ then $\operatorname{dim}(E) \leq \operatorname{dim}_{\xi}(E)$. Let's prove that

$$
\operatorname{dim}_{\xi}(E) \leq \operatorname{dim}(E) .
$$

Fix $t>\operatorname{dim}(E)$ and $\eta>0$ such that $\frac{t}{1+\eta}+\left(2-2(1+\eta)^{3}\right)>\operatorname{dim}(E)$. Let $\varepsilon$ be a positive number such that $\varepsilon \leq \inf _{R \in \xi_{n_{0}}} \operatorname{diam}(R)$. Pick an $\varepsilon$-covering $\left\{R_{j}\right\}_{j}$ of $E$ and set $B_{j}=B\left(x_{j},\left|R_{j}\right|_{M}\right)$ such that $R_{j} \subseteq B_{j}$.

For all $j \in \mathbb{N}$, there exists a family of disjoint rectangles $\left\{R_{j k}\right\}_{k \in L_{j}}$ such that

$$
\bigcup_{k \in L_{j}} R_{j k} \subset B_{j}, \quad P\left(R_{j k}\right) \nsubseteq B_{j} \quad \text { and } \quad B_{j} \subseteq \bigcup_{k \in L_{j}} P\left(R_{j k}\right) .
$$


In a first step, we will calculate the number of $P\left(R_{j k}\right)$ that cover $B_{j}$. We denote by $\lambda$ the Lebesgue measure on $\mathbb{A}_{1} \times \mathbb{A}_{2}$. Using relations (4) and (5), we have

$$
\lambda\left(P\left(R_{j k}\right)\right)^{(1+\eta)^{2}} \leq \lambda\left(R_{j k}\right)
$$

and

$$
\operatorname{diam}\left(B_{j}\right)^{2(1+\eta)^{3}} \leq \lambda\left(R_{j k}\right) .
$$

Let $s$ and $s^{\prime}$ be two positive integers such that

$$
r_{1}^{-(s+1)}<\left|R_{j k}\right|_{M} \leq r_{1}^{-s} \quad \text { and } \quad r_{2}^{-\left(s^{\prime}+1\right)}<\left|R_{j k}\right|_{M} \leq r_{2}^{-s^{\prime}} .
$$

We have

$$
\sum_{k \in L_{j}} \lambda\left(R_{j k}\right) \leq \lambda\left(B_{j}\right) \leq r_{1}^{-s} r_{2}^{-s^{\prime}} \leq\left(r_{1} r_{2}\right) \operatorname{diam}\left(B_{j}\right)^{2}
$$

It follows from inequalities (8) and (9) that

$$
\sum_{k \in L_{j}} \operatorname{diam}\left(B_{j}\right)^{2(1+\eta)^{3}} \leq r_{1} r_{2} \operatorname{diam}\left(B_{j}\right)^{2} .
$$

Hence,

$$
\operatorname{card}\left(L_{j}\right) \leq r_{1} r_{2} \operatorname{diam}\left(B_{j}\right)^{2-2(1+\eta)^{3}} .
$$

In a second step, we have

$$
\left|P\left(R_{j k}\right)\right|_{M}^{1+\eta} \leq\left|R_{j k}\right|_{M} \leq \operatorname{diam}\left(B_{j}\right)
$$

and

$$
\sum_{k \in L_{j}}\left|P\left(R_{j k}\right)\right|_{M}^{t} \leq \sum_{k \in L_{j}} \operatorname{diam}\left(B_{j}\right)^{\frac{t}{1+\eta}}
$$

So,

$$
\begin{aligned}
\sum_{j} \operatorname{diam}\left(R_{j k}\right)^{t} & \leq \sum_{j} \sum_{k \in L_{j}}\left|P\left(R_{j k}\right)\right|_{M}^{t} \\
& \leq \sum_{j}\left(r_{1} r_{2}\right) \operatorname{diam}\left(B_{j}\right)^{2-2(1+\eta)^{3}} \operatorname{diam}\left(B_{j}\right)^{\frac{t}{1+\eta}}
\end{aligned}
$$

and

$$
\mathcal{H}_{\xi, \varepsilon}^{t}(E) \leq\left(r_{1} r_{2}\right) \mathcal{H}_{\varepsilon}^{\frac{t}{1+\eta}+\left(2-2(1+\eta)^{3}\right)}(E)
$$


Letting $\varepsilon$ tend to 0 implies

$$
\mathcal{H}_{\xi}^{t}(E) \leq\left(r_{1} r_{2}\right) \mathcal{H}^{\frac{t}{1+\eta}+2-(1+\eta)^{3}}(E) .
$$

Finally, we obtain

$$
\operatorname{dim}_{\xi}(E) \leq t
$$

And the result yields.

Next, we set a generalization of the Billingsley Theorem [6] in our case. For this purpose, we introduce the following notations. If $E$ is a non empty subset of $\mathbb{A}_{1} \times \mathbb{A}_{2}$ and $x=\left(x_{1}, x_{2}\right) \in E$, let $\xi=\bigcup_{n>1} \xi_{n}$ be a family of rectangles satisfying assumptions (i) and (ii) of Proposition 3.1 and $R_{n}(x)$ be the rectangle of $\xi_{n}$ containing $x$.

In the sequel, we define by $\mathcal{P}\left(\mathbb{A}_{1} \times \mathbb{A}_{2}\right)$ the set of Borel probability measures on $\mathbb{A}_{1} \times \mathbb{A}_{2}$. For all $\mu \in \mathcal{P}\left(\mathbb{A}_{1} \times \mathbb{A}_{2}\right)$ and $\varepsilon>0$, and $E \in \mathbb{A}_{1} \times \mathbb{A}_{2}$, we define

$$
\begin{gathered}
\mu_{\varepsilon}^{\sharp}(E)=\inf \left\{\sum_{j} \mu\left(R_{j}\right): R_{j} \in \xi,\left\{R_{j}\right\}_{j} \text { an } \varepsilon \text {-covering of } E\right\}, \\
\mu^{\sharp}(E)=\lim _{\varepsilon \rightarrow 0} \mu_{\varepsilon}^{\sharp}(E)
\end{gathered}
$$

and

$$
\underset{x \in E, \mu^{\sharp}}{\operatorname{ess} \sup ^{\sharp}} \mathcal{A}(x)=\inf \left\{t \in \mathbb{R}: \mu^{\sharp}(\{x \in E: \mathcal{A}(x)>t\})=0\right\} .
$$

Proposition 3.2. Let $E$ be a subset of $\mathbb{A}_{1} \times \mathbb{A}_{2}$ and $\mu \in \mathcal{P}\left(\mathbb{A}_{1} \times \mathbb{A}_{2}\right)$, we have

(a) $\operatorname{dim}_{\xi}(E) \leq \sup _{x \in E} \liminf _{n \rightarrow \infty} \frac{\log \mu\left(R_{n}(x)\right)}{\log \left(\operatorname{diam}\left(R_{n}(x)\right)\right)} ;$

(b) $\operatorname{Dim}_{\xi}(E) \leq \sup _{x \in E} \limsup _{n \rightarrow \infty} \frac{\log \mu\left(R_{n}(x)\right)}{\log \left(\operatorname{diam}\left(R_{n}(x)\right)\right)}$.

If $\mu^{\sharp}(E)>0$, then we have

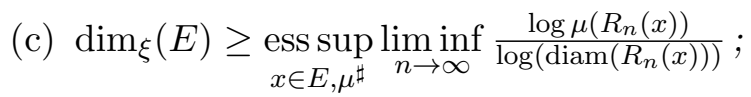

(d) $\operatorname{Dim}_{\xi}(E) \geq \underset{x \in E, \mu^{\sharp}}{\operatorname{ess} \sup _{n \rightarrow \infty}} \limsup _{n \rightarrow \infty} \frac{\log \mu\left(R_{n}(x)\right)}{\log \left(\operatorname{diam}\left(R_{n}(x)\right)\right)}$. 
Proof. Let us prove assumption (a). Take $\delta>\sup _{x \in E} \liminf _{n \rightarrow \infty} \frac{\log \mu\left(R_{n}(x)\right)}{\log \left(\operatorname{diam}\left(R_{n}(x)\right)\right)}$, then for all $x \in E$, there exists $k \geq n$ such that

$$
\mu\left(R_{k}(x)\right) \geq \operatorname{diam}\left(R_{k}(x)\right)^{\delta} .
$$

Let $\varepsilon$ be a positive number, there exists $\left\{R_{j}\right\}_{j}$ a family of pairwise disjoint rectangles such that $E \subset \bigcup_{j} R_{j}$ with

$$
\mu\left(R_{j}\right) \geq \operatorname{diam}\left(R_{j}\right)^{\delta} \quad \text { and } \quad \operatorname{diam}\left(R_{j}\right) \leq \varepsilon .
$$

We have

$$
\sum_{j} \operatorname{diam}\left(R_{j}\right)^{\delta} \leq \sum_{j} \mu\left(R_{j}\right)<\infty
$$

Therefore, $\mathcal{H}_{\xi, \varepsilon}^{\delta}(E)<\infty$. Finally, when $\varepsilon \rightarrow 0$, we get $\operatorname{dim}_{\xi}(E) \leq \delta$ and the result easily follows.

To prove the assumption (b), take $\delta>\sup _{x \in E} \limsup _{n \rightarrow \infty} \frac{\log \mu\left(R_{n}(x)\right)}{\log \left(\operatorname{diam}\left(R_{n}(x)\right)\right)}$. For all $x \in E$, there exists $n \in \mathbb{N}$ such that, for all $k \geq n$ one has

$$
\mu\left(R_{k}(x)\right) \geq \operatorname{diam}\left(R_{k}(x)\right)^{\delta} .
$$

Consider the set

$$
E(n)=\left\{x \in E: \text { for each } k \geq n, \mu\left(R_{k}(x)\right) \geq \operatorname{diam}\left(R_{k}(x)\right)^{\delta}\right\} .
$$

Let $\left\{E_{k}\right\}_{k}$ be a cover of $E$ and $\left\{R_{j}\right\}_{j}$ be an $\varepsilon$-packing of $E(n) \cap E_{k}$ with $\varepsilon<\inf _{R \in \xi_{n_{0}}} \operatorname{diam}(R)$. One has

$$
\sum_{j} \operatorname{diam}\left(R_{j}\right)^{\delta} \leq \sum_{j} \mu\left(R_{j}\right)<\infty .
$$

From which $\overline{\mathcal{P}}_{\xi, \varepsilon}^{\delta}\left(E(n) \cap E_{k}\right)<\infty$. Then we get $\overline{\mathcal{P}}_{\xi}^{\delta}\left(E(n) \cap E_{k}\right)<\infty$ when $\varepsilon \rightarrow 0$. Since $E=\bigcup_{n} E(n)$, we obtain

$$
\operatorname{Dim}_{\xi}(E) \leq \delta .
$$

Hence (b).

and set

Let us prove assumption (c). Take $\delta<\underset{x \in E, \mu^{\sharp}}{\operatorname{ess} \sup } \liminf _{n \rightarrow \infty} \frac{\log \mu\left(R_{n}(x)\right)}{\log \left(\operatorname{diam}\left(R_{n}(x)\right)\right)}$

$$
E_{\delta}=\left\{x \in E: \liminf _{n \rightarrow \infty} \frac{\log \mu\left(R_{n}(x)\right)}{\log \left(\operatorname{diam}\left(R_{n}(x)\right)\right)}>\delta\right\} .
$$


Let

$$
E_{n}=\left\{x \in E_{\delta}: \text { for each } k \geq n, \mu\left(R_{k}(x)\right) \leq \operatorname{diam}\left(R_{k}(x)\right)^{\delta}\right\}
$$

It is clear that $E_{\delta}=\bigcup_{n} E_{n}$. As we have $\mu^{\sharp}\left(E_{\delta}\right)>0$, there exists $n \in \mathbb{N}$ such that $\mu^{\sharp}\left(E_{n}\right)>0$. Then, for any $\varepsilon$-covering $\left\{R_{j}\right\}_{j}$ of $E_{n}$, one has

$$
\mu_{\varepsilon}^{\sharp}\left(E_{n}\right) \leq \sum_{j} \mu\left(R_{j}\right) \leq \sum_{j} \operatorname{diam}\left(R_{j}\right)^{\delta} .
$$

Therefore,

$$
\mu_{\varepsilon}^{\sharp}\left(E_{n}\right) \leq \mathcal{H}_{\xi, \varepsilon}^{\delta}\left(E_{n}\right) .
$$

So,

$$
0<\mu^{\sharp}\left(E_{n}\right) \leq \mathcal{H}_{\xi}^{\delta}\left(E_{n}\right),
$$

which implies

$$
\operatorname{dim}_{\xi}(E) \geq \operatorname{dim}_{\xi}\left(E_{\delta}\right) \geq \operatorname{dim}_{\xi}\left(E_{n}\right) \geq \delta
$$

and assumption (c) yields.

In order to prove assumption (d), let $\delta<\underset{x \in E, \mu^{\sharp}}{\operatorname{ess} \sup _{n \rightarrow \infty}} \limsup _{n \rightarrow \infty} \frac{\log \mu\left(R_{n}(x)\right)}{\log \left(\operatorname{diam}\left(R_{n}(x)\right)\right)}$, and put

$$
E_{\delta}=\left\{x \in E: \limsup _{n \rightarrow \infty} \frac{\log \left(\mu\left(R_{n}(x)\right)\right)}{\log \left(\operatorname{diam}\left(R_{n}(x)\right)\right)}>\delta\right\} .
$$

We have $\mu^{\sharp}\left(E_{\delta}\right)>0$, so there exists a subset $F$ of $E_{\delta}$ such that $\mu^{\sharp}(F)>0$. If $x \in F$, then for all $n \in \mathbb{N}$ there exists $k \geq n$ such that

$$
\mu\left(R_{k}(x)\right) \leq \operatorname{diam}\left(R_{k}(x)\right)^{\delta}
$$

Let $\varepsilon>0$ and $\left\{R_{j}\right\}_{j}$ an $\varepsilon$-packing of $F$ satisfying $(10)$. So,

$$
\mu_{\varepsilon}^{\sharp}(F) \leq \sum_{j} \mu\left(R_{j}\right) \leq \sum_{j} \operatorname{diam}\left(R_{j}\right)^{\delta} .
$$

Then

$$
\mu_{\varepsilon}^{\sharp}(F) \leq \overline{\mathcal{P}}_{\xi, \varepsilon}^{\delta}(F)
$$

This implies

$$
0<\mu^{\sharp}(F) \leq \overline{\mathcal{P}}_{\xi}^{\delta}(F) .
$$


Hence, if $F=\bigcup_{j} F_{j}$, one has

$$
0<\mu^{\sharp}(F)<\sum_{j} \mu^{\sharp}\left(F_{j}\right) \leq \sum_{j} \overline{\mathcal{P}}_{\xi}^{\delta}\left(F_{j}\right) .
$$

Thus,

$$
\mathcal{P}_{\xi}^{\delta}(F)>0
$$

Therefore,

$$
\operatorname{Dim}_{\xi}\left(E_{\delta}\right) \geq \delta,
$$

from which the result follows and we achieve the proof of Proposition 3.2 .

As a consequence of Proposition 3.2, we obtain the following corollary. We adopt the following convention

$$
\frac{\log 0}{\log \rho}=+\infty, \quad \text { for each } \rho>0 .
$$

Corollary 1. Let $\gamma \in \mathbb{R}$. If $\mu$ is a probability Borel measure on $\mathbb{A}_{1} \times \mathbb{A}_{2}$ such that $\mu(E)>0$, we consider a family $\xi$ of rectangles verifying the assumptions of Proposition 3.1 and

$$
E \subset\left\{x \in \mathbb{A}_{1} \times \mathbb{A}_{2}: \lim _{n \rightarrow \infty} \frac{\log \mu\left(R_{n}(x)\right)}{\log \left(\operatorname{diam}\left(R_{n}(x)\right)\right)}=\gamma\right\},
$$

we have

$$
\operatorname{dim}_{\xi}(E)=\operatorname{Dim}_{\xi}(E)=\gamma .
$$

Next, we will be interested in adding an example of application of Corollary 1 .

EXAmple. Let $\left\{\xi_{n}\right\}_{n \geq 1}$ be a sequence of finite partitions of $\mathbb{A}_{1} \times \mathbb{A}_{2}$ made of rectangles in the form $[\omega] \times\left[\omega^{\prime}\right]$, for all $\left(\omega, \omega^{\prime}\right) \in A_{1}^{q(n)} \times A_{2}^{n}$ and $\xi=\bigcup_{n>1} \xi_{n}$, where the integer $q(n)$ is defined such that, for $n \in \mathbb{N}^{*}$

$$
n \frac{\log \left(r_{2}\right)}{\log \left(r_{1}\right)} \leq q(n)<n \frac{\log \left(r_{2}\right)}{\log \left(r_{1}\right)}+1 .
$$

It is clear that the family $\xi$ satisfies the assumptions of Proposition 3.1

For $\alpha \geq 0$, we consider the set

$$
E_{\alpha}=\left\{x \in \mathbb{A}_{1} \times \mathbb{A}_{2}: \lim _{n \rightarrow \infty} \frac{N_{n}^{\omega, \omega^{\prime}}}{n}(x)=\alpha_{\omega, \omega^{\prime}} \text { for all }\left(\omega, \omega^{\prime}\right) \in A_{1} \times A_{2}\right\}
$$


where for $\left(\omega, \omega^{\prime}\right) \in A_{1} \times A_{2}, N_{n}^{\omega, \omega^{\prime}}(x)$ stands for the number of appearances of the couple $\left(\omega, \omega^{\prime}\right)$ in the product word $x_{\mid n} \times y_{\mid n}$ and $\alpha=\left(\alpha_{\omega, \omega^{\prime}}\right)_{\left(\omega, \omega^{\prime}\right) \in A_{1} \times A_{2}}$ is a family of positive numbers such that

$$
\sum_{\left(\omega, \omega^{\prime}\right) \in A_{1} \times A_{2}} \alpha_{\omega, \omega^{\prime}}=1 .
$$

We propose to calculate the Hausdorff dimension of the set $E_{\alpha}$. For this purpose, we consider the Bernoulli measure $\mu$ in $\mathbb{A}_{1} \times \mathbb{A}_{2}$ defined by

$$
\mu\left(\left[\omega_{1} \cdots \omega_{n}\right] \times\left[\omega_{1}^{\prime} \cdots \omega_{n}^{\prime}\right]\right)=\prod_{k=1}^{n} \alpha_{\omega_{k}, \omega_{k}^{\prime}} \quad \text { for each } n \in \mathbb{N}^{*} .
$$

We have

$$
\mu\left(\left[\omega_{1} \cdots \omega_{q(n)}\right] \times\left[\omega_{1}^{\prime} \cdots \omega_{n}^{\prime}\right]\right)=\prod_{k=1}^{n} \alpha_{\omega_{k}, \omega_{k}^{\prime}} \prod_{k=n+1}^{q(n)} \lambda_{\omega_{k}}
$$

with $\lambda_{\omega_{k}}=\sum_{\omega_{k}^{\prime}} \alpha_{\omega_{k}, \omega_{k}^{\prime}}$

It is clear that

$$
E_{\alpha} \subset\left\{x \in \mathbb{A}_{1} \times \mathbb{A}_{2}: \lim _{n \rightarrow \infty} \frac{\log \mu\left(R_{n}(x)\right)}{\log \left(\operatorname{diam}\left(R_{n}(x)\right)\right)}=\gamma\right\},
$$

where

$$
\gamma=-\sum_{\omega, \omega^{\prime}} \frac{\alpha_{\omega, \omega^{\prime}} \log \alpha_{\omega, \omega^{\prime}}}{\log r_{2}}+\left(\frac{1}{\log r_{2}}-\frac{1}{\log r_{1}}\right) \sum_{\omega} \lambda_{\omega} \log \lambda_{\omega}
$$

So, according to the strong law of large numbers we have $\mu\left(E_{\alpha}\right)=1$. By using Corollary 1 we have,

$$
\operatorname{dim}_{\xi}\left(E_{\alpha}\right)=\operatorname{Dim}_{\xi}\left(E_{\alpha}\right)=\gamma
$$

which implies from Proposition 3.1 that

$$
\operatorname{dim}\left(E_{\alpha}\right)=\operatorname{Dim}\left(E_{\alpha}\right)=\gamma .
$$

Thus, any Borel set of $E_{\alpha}$ with dimension inferior to $\gamma$ is of measure $\mu$-zero. 


\section{A VARIANT OF THE REFINED MULTIFRACTAL FORMALISM IN THE PRODUCT SPACE $\mathbb{A}_{1} \times \mathbb{A}_{2}$}

4.1. Problematic. In this section, we will consider a sequence $\left\{\xi_{n}\right\}_{n \geq 1}$ of finite partitions of $\mathbb{A}_{1} \times \mathbb{A}_{2}$ made of rectangles satisfying condition (1) and we put $\xi=\bigcup_{n \geq 1} \xi_{n}$.

In the following, we consider a Borel probability measure $\mu$ on $\mathbb{A}_{1} \times \mathbb{A}_{2}$ and one defines its support $\operatorname{supp}(\mu)$ to be the complement of the set

$$
\bigcup\{R \in \xi: \mu(R)=0\} .
$$

Then, we intend to underestimate the dimensions of the fractal sets $E_{\mu}(\gamma)$ for some values of $\gamma$, where

$$
E_{\mu}(\gamma)=\left\{x \in \operatorname{supp}(\mu): \lim _{n \rightarrow \infty} \frac{\log \mu\left(R_{n}(x)\right)}{\log \left(\operatorname{diam}\left(R_{n}(x)\right)\right)}=\gamma\right\} .
$$

Notice that the natural coverings of these iso-Hölder sets are made of rectangles which become thinner and thinner as their diameter tends to zero which doesn't allow the calculation of the Hausdorff and packing dimensions. For this purpose, we will consider a variant of the refined multifractal formalism as already introduced by F. Ben Nasr and J. Peyrière [5], adapted to rectangles.

Let us consider an auxiliary Borel probability measure $\nu$ on $\mathbb{A}_{1} \times \mathbb{A}_{2}$. If $E$ is a nonempty subset of $\mathbb{A}_{1} \times \mathbb{A}_{2}$ then for $q, t \in \mathbb{R}$ and $\varepsilon>0$, we introduce the following quantities:

$$
\begin{aligned}
\mathbf{H}_{\mu, \nu, \varepsilon}^{q, t}(E) & =\inf \left\{\sum_{j} \mu\left(R_{j}\right)^{q} \operatorname{diam}\left(R_{j}\right)^{t} \nu\left(R_{j}\right):\right. \\
\left.R_{j} \in \xi,\left\{R_{j}\right\}_{j} \text { an } \varepsilon \text {-covering of } E\right\}, & \\
\mathbf{H}_{\mu, \nu}^{q, t}(E) & =\lim _{\varepsilon \rightarrow 0} \mathbf{H}_{\mu, \nu, \varepsilon}^{q, t}(E),
\end{aligned}
$$

and

$$
\begin{aligned}
& \overline{\mathbf{P}}_{\mu, \nu, \varepsilon}^{q, t}(E)=\sup \left\{\sum_{j} \mu\left(R_{j}\right)^{q} \operatorname{diam}\left(R_{j}\right)^{t} \nu\left(R_{j}\right):\right. \\
& \left.R_{j} \in \xi,\left\{R_{j}\right\}_{j} \text { an } \varepsilon \text {-packing of } E\right\}, \\
& \overline{\mathbf{P}}_{\mu, \nu}^{q, t}(E)=\lim _{\varepsilon \rightarrow 0} \overline{\mathbf{P}}_{\mu, \nu, \varepsilon}^{q, t}(E) .
\end{aligned}
$$


The function $\overline{\mathbf{P}}_{\mu, \nu}^{q, t}$ is called the packing pre-measure. In order to deal with an outer measure, one defines

$$
\mathbf{P}_{\mu, \nu}^{q, t}(E)=\inf \left\{\sum_{j} \overline{\mathbf{P}}_{\mu, \nu}^{q, t}\left(E_{j}\right): E \subset \bigcup_{j} E_{j}\right\} .
$$

Let $\varphi$ be the following function

$$
\varphi(q)=\inf \left\{t \in \mathbb{R}: \overline{\mathbf{P}}_{\mu, \nu}^{q, t}(\operatorname{supp}(\mu))=0\right\} .
$$

4.2. Main REsults. Let $\mu$ be a Borel probability measure on $\mathbb{A}_{1} \times \mathbb{A}_{2}$. For $\alpha, \beta \in \mathbb{R}$, one sets

$$
E_{\mu}(\alpha, \beta)=\underline{E}_{\mu}(\alpha) \cap \bar{E}_{\mu}(\beta),
$$

where

$$
\underline{E}_{\mu}(\alpha)=\left\{x \in \operatorname{supp}(\mu): \liminf _{n \rightarrow \infty} \frac{\log \mu\left(R_{n}(x)\right)}{\log \left(\operatorname{diam}\left(R_{n}(x)\right)\right)} \geq \alpha\right\}
$$

and

$$
\bar{E}_{\mu}(\beta)=\left\{x \in \operatorname{supp}(\mu): \limsup _{n \rightarrow \infty} \frac{\log \mu\left(R_{n}(x)\right)}{\log \left(\operatorname{diam}\left(R_{n}(x)\right)\right)} \leq \beta\right\}
$$

Theorem 4.1. Assume that $\varphi(0)=0$ and $\nu^{\sharp}(\operatorname{supp}(\mu))>0$. Then one has

$$
\begin{aligned}
\operatorname{dim}_{\xi} E_{\mu}( & \left.-\varphi_{r}^{\prime}(0),-\varphi_{l}^{\prime}(0)\right) \\
& \geq \inf \left\{\liminf _{n \rightarrow \infty} \frac{\log \nu\left(R_{n}(x)\right)}{\log \left(\operatorname{diam}\left(R_{n}(x)\right)\right)}: x \in E_{\mu}\left(-\varphi_{r}^{\prime}(0),-\varphi_{l}^{\prime}(0)\right)\right\}
\end{aligned}
$$

and

$$
\begin{aligned}
\operatorname{Dim}_{\xi} E_{\mu} & \left(-\varphi_{r}^{\prime}(0),-\varphi_{l}^{\prime}(0)\right) \\
& \geq \inf \left\{\limsup _{n \rightarrow \infty} \frac{\log \nu\left(R_{n}(x)\right)}{\log \left(\operatorname{diam}\left(R_{n}(x)\right)\right)}: x \in E_{\mu}\left(-\varphi_{r}^{\prime}(0),-\varphi_{l}^{\prime}(0)\right)\right\},
\end{aligned}
$$

where $\varphi_{r}^{\prime}, \varphi_{l}^{\prime}$ are respectively the left-hand and right-hand derivatives of $\varphi$. 
Remark 2. The same result holds with

$$
\psi(q)=\inf \left\{t \in \mathbb{R}: \mathbf{P}_{\mu, \nu}^{q, t}(\operatorname{supp}(\mu))=0\right\} .
$$

The proof of Theorem 4.1 is an immediate consequence of the following proposition.

Proposition 4.1. Assume that $\varphi(0)=0$ and $\nu^{\sharp}(\operatorname{supp}(\mu))>0$. Then one has

$$
\nu^{\sharp}\left(E_{\mu}\left(-\varphi_{r}^{\prime}(0),-\varphi_{l}^{\prime}(0)\right)^{c}\right)=0 .
$$

Proof. Take $\delta>-\varphi_{l}^{\prime}(0)$, there exist two positive reals $t$ and $\delta^{\prime}$ such that $\delta>\delta^{\prime}>-\varphi_{l}^{\prime}(0)$ and $\delta^{\prime} t>\varphi(-t)$ which implies $\mathbf{P}_{\mu, \nu}^{-t, \delta^{\prime} t}(\operatorname{supp}(\mu))=0$. So, there exists a partition $\left\{E_{j}\right\}_{j}$ of $\operatorname{supp}(\mu)$ such that

$$
\sum_{j} \overline{\mathbf{P}}_{\mu, \nu}^{-t, \delta^{\prime} t}\left(E_{j}\right) \leq 1
$$

It results that $\overline{\mathbf{P}}_{\mu, \nu}^{-t, \delta t}\left(E_{j}\right)=0$ for all $j$.

Now, consider the set

$$
E_{\delta}=\left\{x \in \operatorname{supp}(\mu): \limsup _{n \rightarrow \infty} \frac{\log \mu\left(R_{n}(x)\right)}{\log \left(\operatorname{diam}\left(R_{n}(x)\right)\right)}>\delta\right\} .
$$

If $x \in E_{\delta}$, for all $n \in \mathbb{N}$ there exists $k \geq n$ such that

$$
\mu\left(R_{k}(x)\right) \leq \operatorname{diam}\left(R_{k}(x)\right)^{\delta} .
$$

Let $E$ be a subset of $E_{\delta}$ and set $F_{j}=E \cap E_{j}$. For $0<\varepsilon \leq \inf _{R \in \xi_{n}} \operatorname{diam}(R)$ and for all $j$, one can find an $\varepsilon$-packing $\left\{R_{j_{k}}\right\}_{k}$ of $F_{j}$ such that

$$
\mu\left(R_{j_{k}}\right) \leq \operatorname{diam}\left(R_{j_{k}}\right)^{\delta} .
$$

So, we have

$$
\begin{aligned}
\nu_{\varepsilon}^{\sharp}\left(F_{j}\right) & \leq \sum_{j} \nu\left(R_{j}\right) \leq \sum_{j} \sum_{k} \nu\left(R_{j_{k}}\right) \\
& \leq \sum_{j} \sum_{k} \mu\left(R_{j_{k}}\right)^{-t} \operatorname{diam}\left(R_{j_{k}}\right)^{\delta t} \nu\left(R_{j_{k}}\right) \leq \sum_{j} \overline{\mathbf{P}}_{\mu, \nu, \varepsilon}^{-t, \delta t}\left(F_{j}\right)=0 .
\end{aligned}
$$


Then

$$
\nu^{\sharp}\left(E_{\delta}\right)=0 .
$$

We conclude that

$$
\nu^{\sharp}\left(\left\{x \in \operatorname{supp}(\mu): \limsup _{n \rightarrow \infty} \frac{\log \mu\left(R_{n}(x)\right)}{\log \left(\operatorname{diam}\left(R_{n}(x)\right)\right)}>-\varphi_{l}^{\prime}(0)\right\}\right)=0 .
$$

In the same way, one proves that

$$
\nu^{\sharp}\left(\left\{x \in \operatorname{supp}(\mu): \liminf _{n \rightarrow \infty} \frac{\log \mu\left(R_{n}(x)\right)}{\log \left(\operatorname{diam}\left(R_{n}(x)\right)\right)}<-\varphi_{r}^{\prime}(0)\right\}\right)=0 .
$$

Proof of Theorem 4.1. Assume that $\varphi(0)=0$ and $\nu^{\sharp}(\operatorname{supp}(\mu))>0$. Then we have according to Proposition 4.1

$$
\nu^{\sharp}\left(E_{\mu}\left(-\varphi_{r}^{\prime}(0),-\varphi_{l}^{\prime}(0)\right)\right)>0 .
$$

So, it is easy to see from Proposition 3.2 that

$$
\operatorname{dim}_{\xi} E_{\mu}\left(-\varphi_{r}^{\prime}(0),-\varphi_{l}^{\prime}(0)\right) \geq \operatorname{ess~sup}_{x \in E_{\mu}\left(-\varphi_{r}^{\prime}(0),-\varphi_{l}^{\prime}(0)\right), \nu^{\sharp}} \liminf _{n \rightarrow \infty} \frac{\log \nu\left(R_{n}(x)\right)}{\log \left(\operatorname{diam}\left(R_{n}(x)\right)\right)},
$$

and

$$
\operatorname{Dim}_{\xi} E_{\mu}\left(-\varphi_{r}^{\prime}(0),-\varphi_{l}^{\prime}(0)\right) \geq \underset{x \in E_{\mu}\left(-\varphi_{r}^{\prime}(0),-\varphi_{l}^{\prime}(0)\right), \nu^{\sharp}}{\operatorname{ess} \sup _{n \rightarrow \infty}} \frac{\log \nu\left(R_{n}(x)\right)}{\log \left(\operatorname{diam}\left(R_{n}(x)\right)\right)} .
$$

However, as a property of ess sup, we know that if $\nu^{\sharp}\left(E_{\mu}\left(-\varphi_{r}^{\prime}(0),-\varphi_{l}^{\prime}(0)\right)\right)$ $>0$, then

$$
\begin{aligned}
\inf _{x \in E_{\mu}\left(-\varphi_{r}^{\prime}(0),-\varphi_{l}^{\prime}(0)\right)} & \left\{\liminf _{n \rightarrow 0} \frac{\log \nu(R(x))}{\log \left(\operatorname{diam}\left(R_{n}(x)\right)\right)}\right\} \\
\leq & \operatorname{ess~sup}_{x \in E_{\mu}\left(-\varphi_{r}^{\prime}(0),-\varphi_{l}^{\prime}(0)\right), \nu^{\sharp}} \liminf _{n \rightarrow \infty} \frac{\log \nu(R(x))}{\log \left(\operatorname{diam}\left(R_{n}(x)\right)\right)}
\end{aligned}
$$

and the proof of the theorem follows. 


\section{An EXAMPLE}

In this section we give a large class of measures satisfying the result of Theorem 4.1. Let $\left\{\xi_{n}\right\}_{n>1}$ be the sequence of finite partitions of $\mathbb{A}_{1} \times \mathbb{A}_{2}$ made of rectangles of the form $[\omega] \times\left[\omega^{\prime}\right]$, for all $\left(\omega, \omega^{\prime}\right) \in A_{1}^{q(n)} \times A_{2}^{n}$ and $\xi=\bigcup_{n \geq 1} \xi_{n}$, where the integer $q(n)$ is defined such that, for $n \in \mathbb{N}^{*}$

$$
n \frac{\log \left(r_{2}\right)}{\log \left(r_{1}\right)} \leq q(n)<n \frac{\log \left(r_{2}\right)}{\log \left(r_{1}\right)}+1 .
$$

For $(i, j) \in A_{1} \times A_{2}$, take $\left(p_{i, j}\right)_{i, j}$ and $\left(q_{i, j}\right)_{i, j}$ two sequences of non negative numbers such that

$$
\sum_{i, j} p_{i, j}=\sum_{i, j} q_{i, j}=1 \quad \text { and } \quad \lambda_{i}=\sum_{j} p_{i, j}=\sum_{j} q_{i, j} .
$$

Let $\left(T_{n}\right)_{n \geq 1}$ be a sequence of integers defined by

$$
T_{1}=1, T_{n}<T_{n+1} \quad \text { and } \quad \lim _{n \rightarrow \infty} \frac{T_{n}}{T_{n+1}}=0 .
$$

Consider the family of parameters $\alpha_{i_{k}, j_{k}}$

$$
\alpha_{i_{k}, j_{k}}= \begin{cases}p_{i_{k}, j_{k}} & \text { if } T_{2 n-1} \leq k<T_{2 n}, \\ q_{i_{k}, j_{k}} & \text { if } T_{2 n} \leq k<T_{2 n+1} .\end{cases}
$$

We define the measure $\mu$ on $\mathbb{A}_{1} \times \mathbb{A}_{2}$ as follows

$$
\mu\left(\left[i_{1} \cdots i_{n}\right] \times\left[j_{1} \cdots j_{n}\right]\right)=\prod_{k=1}^{n} \alpha_{i_{k}, j_{k}} .
$$

It is easy to see that

$$
\mu\left(\left[i_{1} \cdots i_{q(n)}\right] \times\left[j_{1} \cdots j_{n}\right]\right)=\mu\left(\left[i_{1} \cdots i_{n}\right] \times\left[j_{1} \cdots j_{n}\right]\right) \cdot \lambda_{i_{n+1}} \cdots \lambda_{i_{q(n)}} .
$$

In the sequel we will impose those monotony hypotheses

$$
\begin{aligned}
& p_{0,0}<p_{0,1}<\cdots<p_{0, r_{2}-1}<p_{1,0}< \cdots<p_{1, r_{2}-1}<\cdots \\
& \cdots<p_{r_{1}-1,0}<\cdots<p_{r_{1}-1, r_{2}-1}, \\
& q_{0,0}<q_{0,1}<\cdots<q_{0, r_{2}-1}<q_{1,0}< \cdots<q_{1, r_{2}-1}<\cdots \\
& \cdots<q_{r_{1}-1,0}<\cdots<q_{r_{1}-1, r_{2}-1}, \\
& p_{0,0}<q_{0,0} \quad \text { and } \quad p_{r_{1}-1, r_{2}-1}>q_{r_{1}-1, r_{2}-1},
\end{aligned}
$$


which prove the existence of a real $x_{0}$ such that $T\left(x_{0}\right)=W\left(x_{0}\right)$, where

$$
T(x)=\sum_{i, j} \frac{p_{i, j}^{x}}{\sum_{i, j} p_{i, j}^{x}} \log _{r_{2}} p_{i, j}+\left(\frac{1}{\log r_{1}}-\frac{1}{\log r_{2}}\right) \sum_{i, j} \frac{p_{i, j}^{x}}{\sum_{i, j} p_{i, j}^{x}} \log \lambda_{i}
$$

and

$$
W(x)=\sum_{i, j} \frac{q_{i, j}^{x}}{\sum_{i, j} q_{i, j}^{x}} \log _{r_{2}} q_{i, j}+\left(\frac{1}{\log r_{1}}-\frac{1}{\log r_{2}}\right) \sum_{i, j} \frac{q_{i, j}^{x}}{\sum_{i, j}^{x} q_{i, j}^{x}} \log \lambda_{i} .
$$

For this real $x_{0}$, we denote by

$$
\tilde{p}_{i, j}=\frac{p_{i, j}^{x_{0}}}{\sum_{i, j} p_{i, j}^{x_{0}}} \quad \text { and } \quad \tilde{q}_{i, j}=\frac{q_{i, j}^{x_{0}}}{\sum_{i, j} q_{i, j}^{x_{0}}} .
$$

Our aim is to estimate the dimensions of the sets $E_{\mu}(\gamma)$ for certain values of $\gamma$. To be done, we consider an auxiliary measure $\nu$ on $\mathbb{A}_{1} \times \mathbb{A}_{2}$ defined as $\mu$ with the parameters $\tilde{p}_{i, j}$ and $\tilde{q}_{i, j}$ instead of $p_{i, j}$ and $q_{i, j}$ by

$$
\nu\left(\left[i_{1} \cdots i_{n}\right] \times\left[j_{1} \cdots j_{n}\right]\right)=\prod_{k=1}^{n} \tilde{\alpha}_{i_{k}, j_{k}}
$$

where

$$
\tilde{\alpha}_{i_{k}, j_{k}}= \begin{cases}\tilde{p}_{i_{k}, j_{k}} & \text { if } T_{2 n-1} \leq k<T_{2 n}, \\ \tilde{q}_{i_{k}, j_{k}} & \text { if } T_{2 n} \leq k<T_{2 n+1} .\end{cases}
$$

Let $\tilde{\lambda}_{i}=\sum_{j} \tilde{p}_{i, j}=\sum_{j} \tilde{q}_{i, j}$. Then, we have the following result.

Theorem 5.1. For every

$$
\gamma \in\left(-\log _{r_{2}}\left(q_{r_{1}-1, r_{2}-1} \lambda_{r_{1}-1}^{\frac{\log \left(r_{2}\right)}{\log \left(r_{1}\right)}-1}\right),-\log _{r_{2}}\left(q_{0,0} \lambda_{0}^{\frac{\log \left(r_{2}\right)}{\log \left(r_{1}\right)}-1}\right)\right)
$$

we have

$$
\operatorname{dim} E_{\mu}(\gamma) \geq \min \{h(\tilde{p}), h(\tilde{q})\}
$$

and

$$
\operatorname{Dim} E_{\mu}(\gamma) \geq \max \{h(\tilde{p}), h(\tilde{q})\},
$$


where

$$
h(\tilde{p})=-\sum_{i, j} \tilde{p}_{i, j} \log _{r_{2}} \tilde{p}_{i, j}+\left(\frac{1}{\log r_{2}}-\frac{1}{\log r_{1}}\right) \sum_{i} \tilde{\lambda}_{i} \log \tilde{\lambda}_{i}
$$

and

$$
h(\tilde{q})=-\sum_{i, j} \tilde{q}_{i, j} \log _{r_{2}} \tilde{q}_{i, j}+\left(\frac{1}{\log r_{2}}-\frac{1}{\log r_{1}}\right) \sum_{i} \tilde{\lambda}_{i} \log \tilde{\lambda}_{i} .
$$

In order to prove this theorem we will calculate the function $\varphi$ defined in equation (11). For that, we need to use the following lemma.

Lemma 5.1. For $t \in \mathbb{R}$, one has

$$
\varphi(t)=\limsup _{n \rightarrow \infty} \frac{1}{n \log r_{2}} \log \sum_{R_{n} \cap \operatorname{supp}(\mu) \neq \emptyset} \mu\left(R_{n}\right)^{t} \nu\left(R_{n}\right) .
$$

Proof. For $t \in \mathbb{R}$, we denote by

$$
\Phi(t)=\limsup _{n \rightarrow \infty} \frac{1}{n \log r_{2}} \log \sum_{R_{n} \cap \operatorname{supp}(\mu) \neq \emptyset} \mu\left(R_{n}\right)^{t} \nu\left(R_{n}\right) .
$$

We will prove that

$$
\varphi(t)=\Phi(t)
$$

Let's begin by proving that $\varphi(t) \leq \Phi(t)$.

For $\alpha>0$ satisfying $\Phi(t) \leq \alpha$, there exists $n_{0} \in \mathbb{N}$ such that for all $n \geq n_{0}$,

$$
\frac{1}{n \log r_{2}} \log \sum_{R_{n} \cap \operatorname{supp}(\mu) \neq \emptyset} \mu\left(R_{n}\right)^{t} \nu\left(R_{n}\right) \leq \alpha .
$$

So,

$$
\sum_{R_{n} \cap \operatorname{supp}(\mu) \neq \emptyset} \mu\left(R_{n}\right)^{t} \nu\left(R_{n}\right) r_{2}^{-n \alpha} \leq 1, \quad \text { for each } \quad n \geq n_{0} .
$$

Then

$$
\overline{\mathbf{P}}_{\mu, \nu}^{t, \alpha}(\operatorname{supp}(\mu)) \leq 1,
$$

and

$$
\alpha \geq \varphi(t)
$$

which gives that

$$
\Phi(t) \geq \varphi(t)
$$


Next, we prove that $\varphi(t) \geq \Phi(t)$. Let $\alpha>\varphi(t)$, then

$$
\overline{\mathbf{P}}_{\mu, \nu}^{t, \alpha}(\operatorname{supp}(\mu))=0 .
$$

For $\varepsilon>0$, there exists an $\varepsilon$-packing $\left\{R_{n}\right\}_{n}$ of $\operatorname{supp}(\mu)$ such that

$$
\sum_{R_{n} \cap \operatorname{supp}(\mu) \neq \emptyset} \mu\left(R_{n}\right)^{t} \nu\left(R_{n}\right) r_{2}^{-n \alpha} \leq 1 .
$$

Thus

$$
\frac{1}{n \log r_{2}} \log \sum_{R_{n} \cap \operatorname{supp}(\mu) \neq \emptyset} \mu\left(R_{n}\right)^{t} \nu\left(R_{n}\right) \leq \alpha .
$$

So,

$$
\limsup _{n \rightarrow \infty} \frac{1}{n \log r_{2}} \log \sum_{R_{n} \cap \operatorname{supp}(\mu) \neq \emptyset} \mu\left(R_{n}\right)^{t} \nu\left(R_{n}\right) \leq \alpha
$$

and

$$
\Phi(t) \leq \alpha
$$

which prove Lemma 5.1 .

Now, we are able to prove Theorem 5.1. It is easy to see that

$\varphi(t)=\sup \left(\log _{r_{2}} \sum_{i, j} p_{i, j}^{t} \tilde{p}_{i, j}, \log _{r_{2}} \sum_{i, j} q_{i, j}^{t} \tilde{q}_{i, j}\right)+\left(\frac{1}{\log r_{1}}-\frac{1}{\log r_{2}}\right) \log \sum_{i} \lambda_{i}^{t} \tilde{\lambda}_{i}$

and

$$
\varphi(0)=0 .
$$

By the way, using the definitions of the sequences $\left(\tilde{p}_{i, j}\right)$ and $\left(\tilde{q}_{i, j}\right)$ and a simple computation of the derivative of $\varphi$ at 0 we obtain

$$
\varphi^{\prime}(0)=\sum_{i, j} \tilde{p}_{i, j} \log _{r_{2}} p_{i, j}+\left(\frac{1}{\log r_{1}}-\frac{1}{\log r_{2}}\right) \sum_{i} \tilde{\lambda}_{i} \log \lambda_{i} .
$$

Let $\gamma=-\varphi^{\prime}(0)$, it is clear that

$$
\gamma \in\left(-\log _{r_{2}}\left(q_{r_{1}-1, r_{2}-1} \lambda_{r_{1}-1}^{\frac{\log \left(r_{2}\right)}{\log \left(r_{1}\right)}-1}\right),-\log _{r_{2}}\left(q_{0,0} \lambda_{0}^{\frac{\log \left(r_{2}\right)}{\log \left(r_{1}\right)}-1}\right)\right) .
$$


Besides, using the strong law of large numbers we can see that

$$
\liminf _{n \rightarrow \infty} \frac{\log _{r_{2}} \nu\left(R_{n}(x)\right)}{-n}=\min \{h(\tilde{p}), h(\tilde{q})\}
$$

and

$$
\limsup _{n \rightarrow \infty} \frac{\log _{r_{2}} \nu\left(R_{n}(x)\right)}{-n}=\max \{h(\tilde{p}), h(\tilde{q})\},
$$

for $\nu$-almost every $x$.

Then, it follows from Theorem 4.1 and Proposition 3.2 that

$$
\operatorname{dim} E_{\mu}(\gamma) \geq \min \{h(\tilde{p}), h(\tilde{q})\}
$$

and

$$
\operatorname{Dim} E_{\mu}(\gamma) \geq \max \{h(\tilde{p}), h(\tilde{q})\}
$$

which achieve the proof of Theorem 5.1 .

\section{REFERENCES}

[1] N. Attia, B. Selmi, Regularities of multifractal Hewitt-Stromberg measures, Commun. Korean Math. Soc. 34 (2019), 213-230.

[2] N. Attia, B. Selmi, A multifractal formalism for Hewitt-Stromberg measures, J. Geom. Anal. (2019) https://doi.org/10.1007/s12220-019-00302-3.

[3] F. Ben NAsR, I. Bhouri, Spectre multifractal de mesures boréliennes sur $\mathbb{R}^{d}$, C. R. Acad. Sci. Paris Sér. I Math. 325 (1997), 253-256.

[4] F. Ben Nasr, I. Bhouri, Y. Heurteaux, The validity of the multifractal formalism: results and examples, Adv. Math. 165 (2002), 264-284.

[5] F. Ben NasR, J. PeYrière. Revisiting the multifractal analysis of measures, Rev. Mat. Iberoam. 29 (1) (2013), 315-328.

[6] P. Billingsley, "Ergodic Theory and Information", John Wiley \& Sons, Inc., New York-London-Sydney, 1965.

[7] K. Falconer, "Fractal Geometry. Mathematical Foundations and Applications", John Wiley \& Sons, Ltd., Chichester, 1990.

[8] U. Frisch, G. PARISI, Fully developped turbulence and intermittency in turbulence, and predictability in geophysical fluid dynamics and climate dynamics, in "International School of Physics Enrico Fermi, Course 88", (edited by M. Ghil), North Holland, 1985, 84-88.

[9] T.C. Halsey, M.H. Jensen, L.P. Kadanoff, I. Procaccia, B.J. SHRAIMAN, Fractal measures and their singularities: the characterization of strange sets, Phys. Rev. A (3) 33 (1986), 1141-1151.

[10] H.G. Hentschel, I. Procaccia, The infinite number of generalized dimensions of fractals and strange attractors, Phys. D 8 (1983), 435-444. 
[11] B.B. Mandelbrot, Multifractal measures, especially for geophysicist, $A n$ nual Reviews of Materials Sciences. 19 (1989), 514-516.

[12] B.B. MandelBrot, A class of multifractal measures with negative (latent) value for the dimension $f(\alpha)$, in "Fractals: Physical Origin and Properties", in Proceedings of the Special Seminar on Fractals, Erice, 1988 (edited by K. Ford and D. Campbell), American Institute of Physics, 1990, 3-29.

[13] L. Olsen, A multifractal formalism, Adv. Math. 116 (1995), 82-196.

[14] S. SHEN, Multifractal analysis of some inhomogeneous multinomial measures with distinct analytic Olsen's $b$ and B functions, J. Stat. Phys. 159 (5) (2015), $1216-1235$.

[15] C. Tricot, JR., Rarefaction indices, Mathematika 27 (1980), 46-57.

[16] S.J. TAYlor, C. TRICOT, Packing measure, and its evaluation for a Brownian path, Trans. Amer. Math. Soc. 288 (1985), 679-699.

[17] M. Wu, The singularity spectrum $f(\alpha)$ of some Moran fractals, Monatsh. Math. 144 (2005), $141-55$.

[18] M. WU, J. XIAO, The singularity spectrum of some non-regularity Moran fractals, Chaos Solitons Fractals 44 (2011), 548-557.

[19] J. Xiao, M. Wu, The multifractal dimension functions of homogeneous Moran measure, Fractals 16 (2008), 175-185. 